\title{
Controlled Modes Can Be as Effective as CPAP and BiPAP in Non-invasive Ventilation in COVID-19
}

\author{
Abbas Ahmadi (iD ${ }^{1}$, Seyed Yaser Foroghi Ghomi ${ }^{1{ }^{*}}$ and Sarah Lotfi ${ }^{2}$ \\ ${ }^{1}$ Clinical Research Development Center, Shahid Beheshti Hospital, Qom University of Medical Sciences, Qom, Iran \\ ${ }^{2}$ Department of Obstetrics and Gynecology, School of Medicine Nekouei-Hedayati-Forghani Hospital, Qom University of Medical Sciences, Qom, Iran \\ "Corresponding author: Clinical Research Development Center, Shahid Beheshti Hospital, Qom University of Medical Sciences, Qom, Iran. Email: yforoghi@yahoo.com
}

Received 2021 October 16; Revised 2021 October 31; Accepted 2021 November 01

Keywords: Synchronized Intermittent Mandatory Ventilation, Non-invasive Ventilation, COVID-19, Acute Respiratory Failure

\section{Dear Editor,}

Coronavirus disease 2019 (COVID-19) is an infectious disease caused by the newly discovered severe acute respiratory syndrome Coronavirus 2 (1). Non-invasive ventilation (NIV) is the transfer of oxygen (ventilation support) through the face mask; therefore, it can reduce the need for endotracheal intubation (2). The proper and timely use of non-invasive and invasive ventilation in COVID-19 patients can reduce respiratory mortality $(3,4)$. The optimum ventilator support with NIV, indications, modes, and parameters are the issues that can be discussed. This study aimed to prove that when a patient is diagnosed with COVD-19 and is nominated for NIV, other modes, such as synchronized intermittent mandatory ventilation (SIMV), can be used that can make ventilation easier and more effective. Continuous positive airway pressure (CPAP) or bilevel positive airway pressure (BiPAP) is positive airway pressure ventilation in which a constant level of pressure greater than atmospheric pressure is continuously applied to the patient's upper respiratory tract (5). Patients with COVID-19 are usually ventilated primarily by this mode that is the most popular ventilation method $(6,7)$.

Controlled modes can be as effective as CPAP and BiPAP in NIV in COVID-19, which is why SIMV mode is recommended among the primary modes, such as CPAP or BiPAP. The SIMV is a type of volume or pressure control mode of ventilation. By the utilization of this model, the ventilator performs several forced breaths (sets) with the volume adjusted and at the same time makes spontaneous breathing possible $(8,9)$. The SIMV has the benefits of avoiding acute respiratory alkalosis and allows patients to achieve normal alveolar ventilation through a healthy ventilation drive (10). This study used SIMV in these patients and made oxygenation and blood gas exchange more efficient. Other invasive approaches were also important in COVID-19 patients (11). Therefore, further clinical studies are recommended in this regard.

\section{Footnotes}

Authors' Contribution: A.A. developed the original idea and the protocol, abstracted and analyzed the data, wrote the manuscript, and was the guarantor. S.F. and S.L. contributed to the development of the protocol, abstracted the data, and prepared the manuscript.

Conflict of Interests: The authors declare that there is no potential conflict of interest in this manuscript.

Funding/Support: It was not declared by the authors.

\section{References}

1. Pergolizzi JV, Magnusson P, LeQuang JA, Breve F, Paladini A, Rekatsina $\mathrm{M}$, et al. The Current Clinically Relevant Findings on COVID-19 Pandemic. Anesth Pain Med.2020;10(2). e103819. doi: 10.5812/aapm.103819. [PubMed: 32754437]. [PubMed Central: PMC7352949].

2. Nava S, Hill N. Non-invasive ventilation in acute respiratory failure. Lancet. 2009;374(9685):250-9. doi:10.1016/S0140-6736(09)604967. [PubMed: 19616722]. [PubMed Central: PMC7138083].

3. Rahimzadeh P, Faiz HR, Farahmandrad R, Hassanlouei B, Habibi A, Hedayati Emami S, et al. Clinical Features and Prognosis of Invasive Ventilation in Hospitalized Patients with COVID-19: A Retrospective Study. Anesth Pain Med. 2020;10(6). e108773. doi: 10.5812/aapm.108773. [PubMed: 34150575]. [PubMed Central: PMC8207877].

4. Mahmoodpoor A, Shadvar K, Ghamari AA, Mohammadzadeh Lameh M, Asghari Ardebili R, Hamidi M, et al. Management of Critically Ill Patients with COVID-19: What We Learned and What We Do. Anesth Pain Med. 2020;10(3). e104900. doi: 10.5812/aapm.104900. [PubMed: 32944565]. [PubMed Central: PMC7472789].

5. Haryalchi K, Heidarzadeh A, Abedinzade M, Olangian-Tehrani S, Ghazanfar Tehran S. The Importance of Happy Hypoxemia in COVID-19. Anesth Pain Med. 2021;11(1). e111872. doi: 10.5812/aapm.111872. [PubMed: 34221943]. [PubMed Central: PMC8239260]. 
6. Kafan S, Tadbir Vajargah K, Sheikhvatan M, Tabrizi G, Salimzadeh A, Montazeri M, et al. Predicting Risk Score for Mechanical Ventilation in Hospitalized Adult Patients Suffering from COVID-19. Anesth Pain Med.2021;11(2). e112424. doi:10.5812/aapm.112424. [PubMed:34336617]. [PubMed Central: PMC8314086].

7. Rahimzadeh P, Amniati S, Farahmandrad R, Faiz SHR, Hedayati Emami S, Habibi A. Clinical Characteristics of Critically Ill Patients Infected with COVID-19 in Rasoul Akram Hospital in Iran: A Single Center Study. Anesth Pain Med. 2020;10(5). e107211. doi: 10.5812/aapm.107211. [PubMed: 34150569]. [PubMed Central: PMC8207836].

8. Lazoff SA, Bird K. Synchronized Intermittent Mandatory Ventilation. Treasure Island, USA: StatPearls; 2021.

9. Mohammadi M, Khafaee Pour Khamseh A, Varpaei HA. Invasive Air- way "Intubation" in COVID-19 Patients; Statistics, Causes, and Recommendations: A Review Article. Anesth Pain Med. 2021;11(3). e115868. doi: 10.5812/aapm.115868. [PubMed: 34540642]. [PubMed Central: PMC8438719].

10. Hudson LD, Hurlow RS, Craig KC, Pierson DJ. Does intermittent mandatory ventilation correct respiratory alkalosis in patients receiving assisted mechanical ventilation? Am Rev Respir Dis 1985;132(5):1071-4. doi: 10.1164/arrd.1985.132.5.1071. [PubMed: 3933391].

11. Hassani V, Amniati S, Ahmadi A, Mohseni M, Sehat-Kashani S, Nikoubakht N, et al. Emergency Tracheostomy in Two Airway Trauma Patients Suspected of COVID-19: A Case Report. Anesth Pain Med. 2020;10(4). e104648. doi: 10.5812/aapm.104648. [PubMed: 33134149]. [PubMed Central: PMC7539045]. 